\title{
Welcome on Behalf of the Wiki Workshop Chairs
}

It is our great pleasure to welcome you to the Wiki Workshop at the Web Conference 2018. The goal of this workshop is to bring together researchers exploring all aspects of Wikimedia projects such as Wikipedia, Wikidata, and Wikimedia Commons. With members of the Wikimedia Foundation's Research team on the organizing committee and with the experience of successful workshops in 2015, 2016, and 2017, we aim to continue facilitating a direct pathway for exchanging ideas between the organization that coordinates Wikimedia projects and the researchers interested in studying them.

We received 17 paper submissions (and counting) from all around the world covering a broad range of topics. Our program committee evaluated them regarding relevance, quality, and novelty, selecting 8 to be presented as posters and published in the workshop proceedings, and numerous others for poster presentation only.

In addition to these contributed posters, we are looking forward to hosting 6 high-profile invited speakers (in alphabetical order):

- Andrea Forte, Associate Professor at Drexel University

- Jon Kleinberg, Professor at Cornell University

- Markus Krötzsch, Professor at Technical University of Dresden

- Katherine Maher, Executive Director at the Wikimedia Foundation

- Gerhard Weikum, Research Director at the Max Planck Institute for Informatics

- Cong Yu, Research Scientist at Google Research, New York City

We hope that you will join us for these speakers' presentations and for the lively discussions that are certain to ensue around the most pressing issues and the most promising opportunities faced by Wikipedia, Wikidata, and other Wikimedia projects.

$\begin{array}{ll}\begin{array}{ll}\text { Robert West } \\ \text { Workshop Co-chair }\end{array} & \begin{array}{l}\text { Leilla Zia } \\ \text { WPFL }\end{array} \\ \begin{array}{ll}\text { Wikimedia Foundation } \\ \text { Dario Taraborelli }\end{array} & \begin{array}{l}\text { Jure Leskovec } \\ \text { Workshop Co-chair }\end{array} \\ \begin{array}{l}\text { Wikimedia Foundation } \\ \text { Worksop Co-chair }\end{array} & \text { Stanford University }\end{array}$

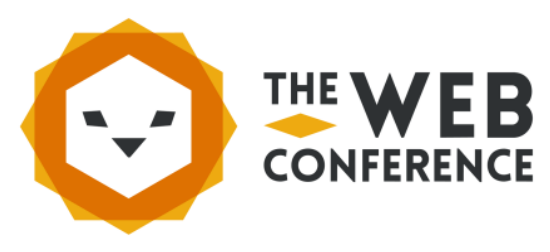




\section{Wiki Workshop Organization}

Co-Chairs: Robert West, EPFL

Leila Zia, Wikimedia Foundation

Dario Taraborelli, Wikimedia Foundation

Jure Leskovec, Stanford University

Program Committee: Michele Catasta, Stanford University

Jérôme Hergueux, ETHZ

Srijan Kumar, Stanford University

Florian Lemmerich, RWTH Aachen University

Jonathan Morgan, Wikimedia Foundation

Tiziano Piccardi, EPFL

Miriam Redi, Wikimedia Foundation

Diego Saez-Trumper, Wikimedia Foundation

Nithum Thain, Jigsaw

Morten Warncke-Wang, University of Minnesota 